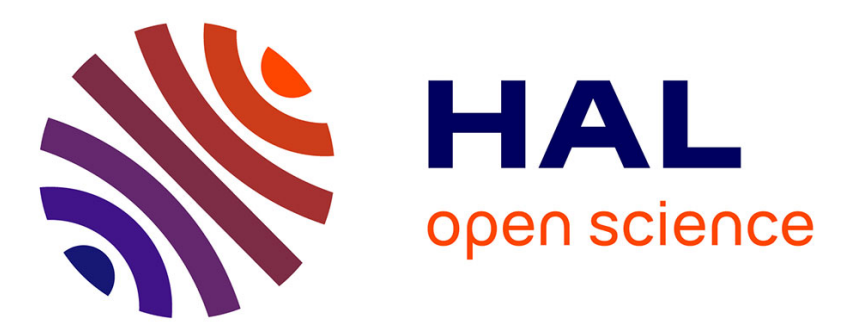

\title{
Nodes Placement for reducing Energy Consumption in Multimedia Transmissions
}

\author{
Pasquale Pace, Valeria Loscrì, Enrico Natalizio, Tahiry Razafindralambo
}

\section{To cite this version:}

Pasquale Pace, Valeria Loscrì, Enrico Natalizio, Tahiry Razafindralambo. Nodes Placement for reducing Energy Consumption in Multimedia Transmissions. 22nd IEEE Symposium on Personal, Indoor, Mobile and Radio Communications (PIMRC), Sep 2011, Toronto, Canada. inria-00602321

\section{HAL Id: inria-00602321 \\ https://hal.inria.fr/inria-00602321}

Submitted on 12 Dec 2013

HAL is a multi-disciplinary open access archive for the deposit and dissemination of scientific research documents, whether they are published or not. The documents may come from teaching and research institutions in France or abroad, or from public or private research centers.
L'archive ouverte pluridisciplinaire HAL, est destinée au dépôt et à la diffusion de documents scientifiques de niveau recherche, publiés ou non, émanant des établissements d'enseignement et de recherche français ou étrangers, des laboratoires publics ou privés. 


\section{Nodes Placement for reducing Energy Consumption in Multimedia Transmissions}

\author{
Pasquale Pace Valeria Loscrí \\ DEIS - University of Calabria Rende, ITALY \\ \{ppace, vloscri\}@deis.unical.it
}

\author{
Enrico Natalizio Tahiry Razafindralambo \\ INRIA Lille - Nord Europe, FRANCE \\ \{enrico.natalizio, tahiry.razafindralambo\}@inria.fr
}

\begin{abstract}
Power consumption is an essential issue in wireless multimedia sensor networks (WMSNs) due to the elevated processing capabilities requested by the video acquisition hardware installed on the generic sensor node. Hence, node placement scheme in WMSNs greatly impacts the overall network lifetime. In this context, the paper first proposes a suitable hardware architecture to implement a feasible WMS node based on off-the-shelf technology, then it shows how the energy spaced placement of the wireless nodes reduces the energy consumption in comparison with the classical evenly spaced placement, without affecting the video quality of multimedia traffic.
\end{abstract}

Index Terms-Wireless Sensor Networks, Multimedia, Energy Saving, Quality of Experience.

\section{INTRODUCTION}

A wireless sensor network (WSN) is a collection of small and constrained devices that communicate via wireless links in a multihop fashion. In recent years, the research on wireless sensor networks has received a lot of attentions, due to their potential applications in various areas such as emergency/rescue operations, military operations, environment monitoring [1], [2].

In most of the deployments, sensors are expected to send few amount of data (temperature, humidity, etc.) either regularly or upon the detection of a specific event to a central entity, called sink (or base station). Due to resource limitation, the base station may not be reachable directly by each sensor. Thanks to the wireless communication capabilities and the protocols developed in the literature, multi-hop transmissions can be used to route data from a sensor to the sink when no direct connection is available. Moreover, sensors are supposed to be static or quasi-static.

Recently, a new generation of sensors appeared. These sensors have much more capabilities than the low-capacity (processor, memories, battery) sensors used so far. They are equipped with low cost cameras and/or microphones to be able to acquire and transmit multimedia contents to the base station. The multimedia sensing capabilities, provided by this new generation of sensors, raise some new issues in the field of wireless sensor networks due to their uniqueness [3],[4]. These new sensors produce a huge amount of data with Quality of Service (QoS) restrictions and requirements such as end-toend delay. Furthermore, constraints such as energy, delay, loss ratio and throughput are still an issue.
Dimensioning a multimedia sensor network should be done by anticipating users' needs and issues. Unfortunately, this is not always feasible. It is not easy to increase wireless link capacity or performance (delay, bandwidth, etc.) since sensors and wireless links are finite resources. In the context of wireless networks and wireless multimedia networks, Akyildiz et al. stated in Elsevier Computer Communication 2007 [5]:

"[...] capacity and delay attainable on each link are location dependent, vary continuously, and may be bursty in nature, thus, making quality of service (QoS) provisioning a challenging task [...]"

In this paper, we evaluate the quality of service provided at the application layer for a multimedia traffic. We show how nodes' placement can impact the performance by increasing or decreasing the multimedia quality. We use a video evaluation framework to extract multimedia metrics such as MOS (Mean Opinion Score)[6], VQM (Video Quality Meter)[7] and try different node placements (energy spaced, evenly spaced) to show that small differences in the placement $( \pm 7 \%$ in distance) have a remarkable impact on the energy saving by extending the lifetime of the network up to more than $15 \%$ while preserving video quality.

The rest of the paper is organized as follow. Section II gives the state of the art of sensor placement schemes for multimedia traffic. Section III describes the placement schemes used for the evaluation in this paper. Section IV is devoted to the system model. Simulation results are provided in Section V before concluding the paper in Section VI.

\section{RELATED WORKS}

The placement of the nodes can highly affect the performance of the network in terms of routing, capacity, delay, throughput, energy consumption, etc. [5], [8]. At the best of our knowledge, no works have been proposed to study the effect of nodes placements on both the quality of a multimedia transmission and the energy consumption of the network.

The work proposed by So et al in [9], shows the effect of relay nodes' placement in wireless local area networks. The authors study how the number of relay nodes and the characteristics of the traffic affect the optimal relay node placement and the expected throughput capacity of the network. The authors do not give any outline on how to place the nodes to increase the capacity or reduce the energy consumption. 
In [10], the authors show the effect of node placement in ultra wideband sensor networks by evaluating the performances of communication reliability at the MAC layer.

Cao et al. in [8] make a comparison among placements of nodes on the straight line between source and destination, but they do not consider any multimedia aspect.

\section{Placements USed}

In this work we consider two schemes of placement. The first is referred as Evenly or Uniform placement and is taken from [11]. Specifically, in [11], authors show that the best configuration for a monodirectional data flow in terms of energy consumption is to place relay nodes in the evenly spaced positions on the straight line between the source and the destination node. This result is based on the intrinsic assumption that nodes have all the same residual energy. Once a more realistic assumption is taken into consideration, namely nodes can have different residual energies, the best placement for the energy consumption is not anymore the evenly spaced but the energy spaced [12]. Specifically, authors derive a close formula to mathematically compute the position of each node depending on the residual energy of the nodes involved in the data flow, which we recall in (1):

$$
\mathbf{v}_{i}=\mathbf{v}_{1}+\sum_{k=1}^{i-1} \sqrt{\frac{E_{k}}{P_{r e c} T_{P L}}} \mathbf{u}, i=2, \ldots, n-1 .
$$

where the position of node $i\left(v_{i}\right)$ is determined incrementally from the positions of all the previous nodes by taking into consideration their residual energy. An inherent property of the placement considered in [12] is that nodes relaying the same data flow will completely deplete their batteries all in the same moment. In fact, from (1), we have that the pathlifetime can be found from

$$
T_{P L}=\frac{1}{P_{r e c}\left\|\mathbf{v}_{n}-\mathbf{v}_{1}\right\|^{2}}\left(\sum_{i=1}^{n-1} \sqrt{E_{i}}\right)^{2}
$$

and the following property is verified:

$$
T_{P L}=T_{1}=T_{2}=\cdots=T_{n-1} .
$$

This is a very interesting property in wireless sensor networks, since when the batteries of nodes have different durations, the first node that dies affects the whole data flow. In fact, energy will be wasted to send control packets in order to build a new path or to restore the previous one. Moreover, before being notified that the path is broken, the source will keep sending packets on the broken path causing many packet losses. In this work, if we consider the specific characteristics of multimedia data that we want to transmit, the choice of a similar placement seems to be suitable for the purpose. In Fig. 1 we show the two placements. Even if the positions of nodes are similar for the two schemes, in Section V we will show the impact of the different placement on the quality of the multimedia transmission and the energy consumption.

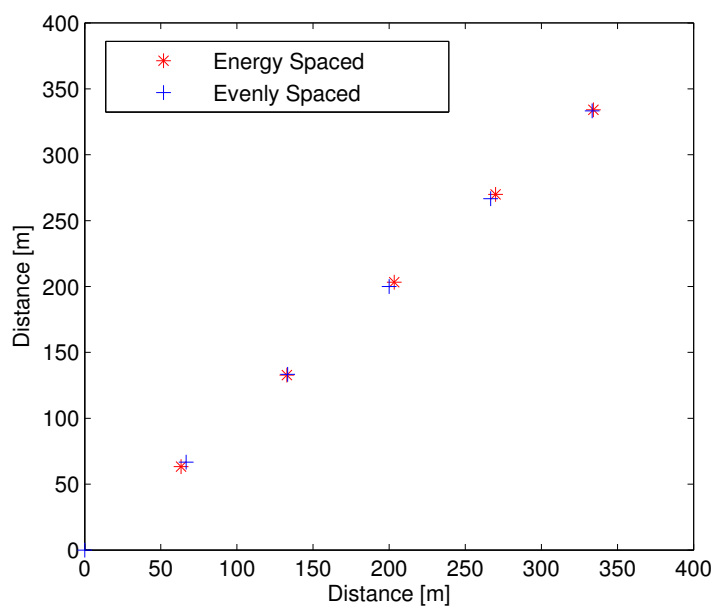

Fig. 1. Nodes' positions with the Energy and Evenly placements.

\section{SYSTEM MODEL}

\section{A. Hardware choices and energy consumption evaluation}

The main objective of this work consists in evaluating the energy consumption of video transmissions in MWSN by using different placements. Hence, a feasible hardware implementation of the generic wireless multimedia sensor node will be detailed in this section by illustrating the specific energy consumption of each module of the sensor node. This analysis is mainly finalized to discover the "realistic" values of energy absorbed by the hardware components, in order to use them during the simulation phase. In particular, we propose to design a multimedia sensor node constituted by two different modules: a new low energy wireless transmitter called WiFly GX [13] based on WiFi standard (fig.2.a), and a well known $C M U$ cam 3 video camera (fig.2.b) able to send CIF files format $(352 \times 288$ pixels $)$ [14]. The two modules can work together once mounted on top of a four lithium cell AA batteries.

Specifically:

- the WiFly $G X$ is perfect for mobile wireless applications such as asset monitoring, GPS tracking and portable devices because of its small size and extremely low power consumption;

- the $C M U$ cam3 implements a fully programmable embedded computer vision sensor and it guarantees a good video quality for light applications.

An additional motivation for us to use these hardware modules to implement a MWS node is given by the fact that they represent a well documented off-the-shelf technology. They can assess the average energy consumption in terms of transmission power, receiver sensitivity and supply voltage as resumed in Table I.

Regarding the initial maximum energy value, each MWS node can be equipped with four AA batteries of $1100 \mathrm{mAh}$ that generate an initial energy equal to $23760 \mathrm{Joule}$. To complete our consumption analysis, we need to take into account a 


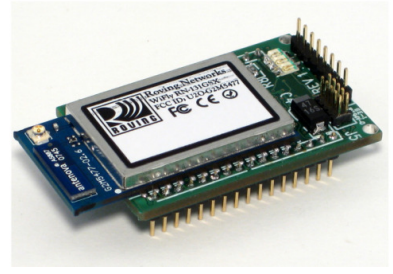

(a)

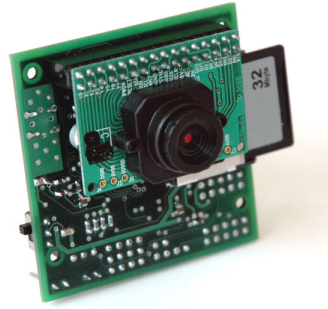

(b)
Fig. 2. Hardware modules of a generic MWS node: a) WiFly GX and b) CMUCam3.

TABLE I

MWS NODE HARDWARE CHARACTERISTICS

\begin{tabular}{|c|c|}
\hline \multicolumn{2}{|c|}{ Radio Characteristics of WiFly GX module } \\
\hline Receive sensitivity & $-85 \mathrm{dBm}$ \\
\hline Antenna TX Power & $18 \mathrm{dBm}=63.09 \mathrm{~mW}$ \\
\hline \multicolumn{2}{|c|}{ Electrical Characteristics of WiFly GX module } \\
\hline Supply Voltage & {$[2-3.3] V$} \\
\hline \multicolumn{2}{|c|}{ WiFly GX Power Consumption } \\
\hline Standby/Idle & $35 m A$ \\
\hline Connected (RX) & $40 m A$ \\
\hline Connected (TX) & $110 m A$ \\
\hline \multicolumn{2}{|c|}{ CMUcam3 General Characteristics } \\
\hline max resolution & CIF $352 \times 288$ \\
\hline color depth & 8bits per pixel \\
\hline frame rate & 26FPS \\
\hline \multicolumn{2}{|c|}{ CMUcam3 Power Consumption [15] } \\
\hline Active Cam & $500 \mathrm{~mW}$ \\
\hline Disable Cam & $300 \mathrm{~mW}$ \\
\hline
\end{tabular}

TABLE II

MWS NODE OVERALL POWER CONSUMPTION

\begin{tabular}{|c|c|}
\hline TXPower $_{S}=T X_{\text {antenna }}+T X_{W i F l y}+$ active $_{C a m}$ & $0.893 W$ \\
\hline TXPower $_{R}=$ TX $_{\text {antenna }}+$ TX $_{W i F l y}+$ disable $_{\text {Cam }}$ & $0.693 W$ \\
\hline RXPower $=R X_{\text {WiFly }}+$ disable $_{C a m}$ & $0.420 W$ \\
\hline IdlePower $=$ Idle $_{W i F l y}+$ disable $_{\text {Cam }}$ & $0.405 \mathrm{~W}$ \\
\hline CSThresh $=$ receive Sensitivity $=-85 \mathrm{dBm}$ & $3.162 e^{-12} W$ \\
\hline RXThresh (transmission range $100 \mathrm{~m}$ ) & $6.232 e^{-10} W$ \\
\hline
\end{tabular}

supply voltage of $3 \mathrm{~V}$ that turns into the following power consumptions for the WiFly GX module:

$$
\begin{aligned}
& T_{X P^{-}} \text {Power }_{W l y}=110 \mathrm{~mA} * 3 \mathrm{~V}=330 \mathrm{~mW} \\
& R X \text { Power }_{W i F l y}=40 \mathrm{~mA} * 3 \mathrm{~V}=120 \mathrm{~mW} \\
& \text { IdlePower }_{W i F l y}=35 \mathrm{~mA} * 3 \mathrm{~V}=105 \mathrm{~mW}
\end{aligned}
$$

Mixing together all the operating parameters discussed so far, we can obtain the overall consumption values for the proposed MWS node architecture as shown in Table II. In the Table $T X$ Power $_{S}$ represents the transmission power consumption of the source node whilst $T X$ Power $_{R}$ is the transmission power consumption of the generic relay or destination node in which the CMUCam 3 module is in the disable state.

\section{B. Simulation Framework and Multimedia Traffic}

Upon the determination of the energy consumption parameters for feasible MWS nodes, we used these parameters in the simulation of different placement strategies by using EvalVid [16]. EvalVid is a complete framework and tool-set for quality evaluation of videos transmitted over a real or simulated communication network. This framework is able to measure QoS parameters of the underlying network, such as packet loss, delays and jitter. Moreover, it supports a subjective video quality evaluation of the received video based on the frame-by-frame PSNR (Peak Signal Noise Ratio) calculation. It has a modular and network independent structure, which permits all the interactions with the network via several trace files. EvalVid can be combined with the well known NS2 simulator in order to obtain a robust and reliable framework for evaluating the perceived quality of service for multimedia video content.

Concerning the multimedia traffic, we chose to use four different video sequences of commonly used video test contents in the 4:2:0 YUV format [17]. These video sequences have the same CIF resolution, which is compatible with the presented hardware, and different properties in terms of data rate and change of scene. In particular, we coded the uncompressed YUV files using an MPEG-4 codec in order to create compressed raw videos with different data rate as shown in figure 3.

\section{PQoS evaluation: PSNR and VQM}

Since MWSNs are well suited for video content provisioning, the best way to evaluate the system performance consists in using video quality indexes based on human vision and real PQoS (Perceived Quality of Service) or QoE (Quality of Experience). Over the last years, emphasis has been put on developing methods and techniques for evaluating the perceived quality of digital video content. These methods are mainly categorized into two classes: subjective and objective.

The subjective test methods involve an audience of people, who watch a video sequence and score its quality as perceived by them, under specific and controlled watching conditions. The arithmetic mean of all the opinion scores collected is the MOS (Mean Opinion Score) standardized by ITU-T [6]. Since the preparation and execution of subjective methods is costly and time consuming, researchers have turned to simple error measures such as MSE (Mean-Squared Error) or PSNR, suggesting that they would be equally valid.

The EvalVid tool integrates the module for computing the PSNR value of a transmitted video, so that we can use this feature to evaluate the goodness of the multimedia transmission according to different nodes placements. Moreover, according to the work conducted in [18] it is possible to match few ranges of PSNR values with the corresponding MOS index in order to use both subjective and objective quality metrics (see Table IV).

Unfortunately, the PSNR metric does not take into account human vision; thus, it cannot be a reliable predictor of perceived visual quality because this simple objective measure 


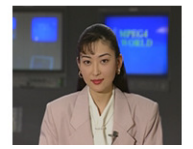

Akiyo

581 kbps

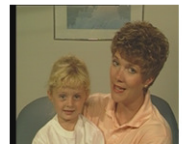

Mother-Daughter 1027 kbps

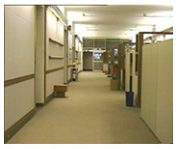

Hall $2939 \mathrm{kbps}$

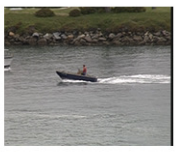

Coastguard $4738 \mathrm{kbps}$
Fig. 3. Multimedia Video Contents and Average Data Rates.

TABLE III

SIMULATION PARAMETERS

\begin{tabular}{|c|c|}
\hline Field Area $(L \times L)$ & $400 \mathrm{~m} \times 400 \mathrm{~m}$ \\
\hline Source Position $\left(x_{S}, y_{S}\right)$ & $(0,0)$ \\
\hline Destination Position $\left(x_{D}, y_{D}\right)$ & $(400,400)$ \\
\hline Initial Maximum Residual Energy $\left(E_{\max }\right)$ & $23760 \mathrm{~J}$ \\
\hline Initial Minimum Residual Energy $\left(E_{\min }\right)$ & $80 \%$ of $E_{\max }$ \\
\hline Maximum Transmission Range $(r)$ & $100 \mathrm{~m}$ \\
\hline Transmission Rate $\left(r_{T}\right)$ & $1,2,5.5,11 \mathrm{Mb} / \mathrm{s}$ \\
\hline Actual Videos Transmission Rate $\left(r v_{T}\right)$ & see figure 3 \\
\hline Propagation Error-Free Model & Two-Ray ground \\
\hline MAC Protocol & Standard $802.11 \mathrm{~b}$ \\
\hline Routing Protocol & AODV \\
\hline Number of run for each scenario & 100 \\
\hline Interval confidence for average & $95 \%$ \\
\hline
\end{tabular}

operates solely on the basis of pixel-wise differences neglecting the impact of viewing conditions on the actual visibility of videos. For this reason, in our study, we also considered the VQM (Video Quality Meter) index that represents a more general and robust purpose video quality model taking into account specific issues of human vision [7] [19].

\section{RESUlTS}

In this section, we will show the results obtained by simulating the behavior of the two aforementioned nodes placements in the integrated simulation framework EvalVid+NS2, when multimedia data has to be delivered from a source node to a destination node. In Table III, we reported the values of the parameters used throughout the simulation.

The performance parameters considered in this work are: PSNR and VQM as explained in section IV-C. Furthermore, since the main objective of the paper is to investigate the energy consumption issue of nodes when they relay multimedia traffic, we also consider the lifetime of the network.

\section{A. Simulation Campaigns}

1) Nodes Number: A first simulation campaign has been conducted to determine the impact of the number of relay nodes on the measured PSRN. The placement used in this first simulation campaign is the evenly spaced. In Fig. 4 we reported the value of PSNR when the number of nodes involved in the multimedia transmission increases. With the aim of highlighting the decrease in video quality due to multihop, the basic configuration is firstly constituted only by source and destination, progressively the number of nodes increases from 2 to 7 and, consequently, the number of relay nodes from 0 to 5 . Since in our simulations the distance between nodes is kept constant, the field area dimension has
TABLE IV

PSNR AND VIDEO QUALITY

\begin{tabular}{|c|c|}
\hline PSNR Value & MOS Value (Quality) \\
\hline$>37 d B$ & 5 (Excellent) \\
$31 \div 37 d B$ & 4 (Good) \\
$25 \div 31 d B$ & 3 (Fair) \\
$20 \div 25 d B$ & 2 (Poor) \\
$<20 d B$ & 1 (Bad) \\
\hline
\end{tabular}

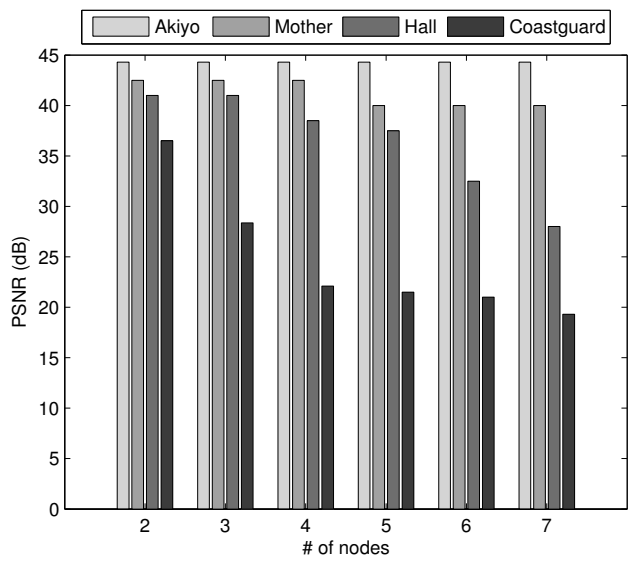

Fig. 4. PSNR when number of nodes varies in the transmission of the 4 videos of Figure 3.

been increased accordingly from $66 \mathrm{~m} \times 66 \mathrm{~m}$ considering only two nodes, to $400 \mathrm{~m} \times 400 \mathrm{~m}$ for the configuration with seven evenly spaced nodes.

In Table IV, we recalled the values used to classify the quality of a video through the usage of the PSNR metric. We can see that the number of nodes involved in the flow does not affect the transmission of the videos with the two lowest bit rates, whose quality is always excellent. In this case, the capacity of the channel is large enough to permit a very high quality transmission. In order to appreciate the effect of a multihop transmission for such low bit rate videos, we will investigate the behavior of the placements for multiflow transmission as future works. When the bit rate of the transmitted video increases and saturates the capacity of the channel, the quality decreases drastically, especially for a higher number of nodes. In fact, the quality of "Hall" degrades from excellent to fair, and that of "Coastguard" from good to bad in the longest multihop path. If we pay particular attention to this last video we can argue that, even if the difference in video quality between the scenario with 4 nodes and the one with 7 nodes is very small (i.e., about $3 \mathrm{~dB}$ ), the high transmission rate required by this specific video, makes the fruition of the video content almost impossible in extended multihop scenarios.

In the rest of the simulation we will use the scenario with 7 nodes.

2) Comparison of placements: The first comparison that we propose regards again the value of PSNR for both energy 


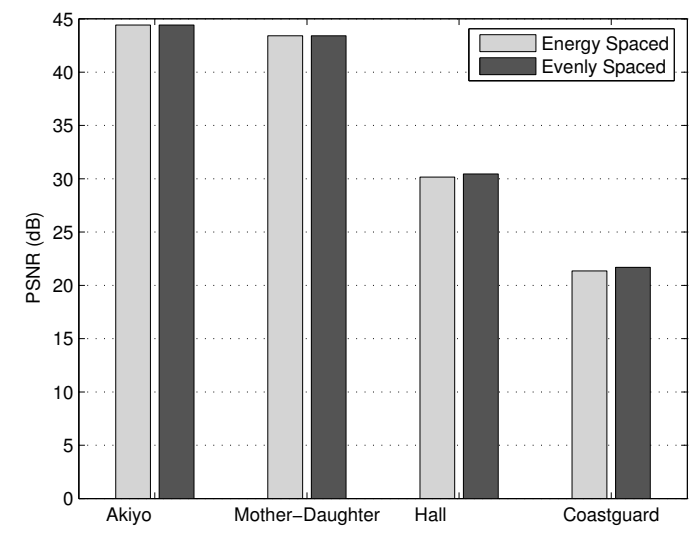

Fig. 5. PSNR achieved by the two different placements for the scenario with 7 nodes.

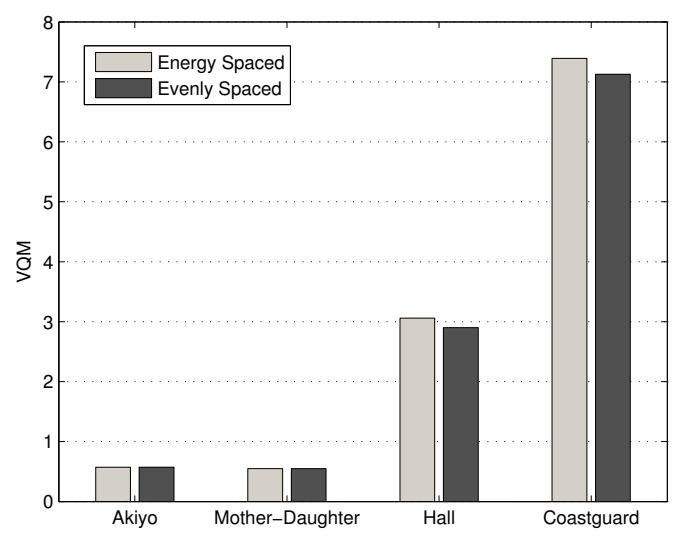

Fig. 6. VQM achieved by the two different placements.

spaced placement and evenly spaced placement. In fact, it is important to notice that the energy spaced placement has been introduced in order to face the issue of energy consumption. Therefore, it could show a bad behavior in terms of video quality in respect of a more evenly distributed placement.

In Fig. 5 we show that, even if relay nodes are placed in different positions, the performance of the two placement schemes in terms of PSNR is the same. In fact, both the schemes obtain excellent quality for the two lowest bit rate videos, whereas they incur in a fair and a poor quality for the highest bit rate videos, respectively.

Since the only valuable information that was possible to extract from the previous plot was that the energy spaced placement obtains the same results of the evenly spaced in terms of PSNR, we decided to compare the two placements schemes according to the metrics explained in Section IV-C. As we said in the mentioned Section, the VQM is a more precise index of the quality perceived by the observer. VQM has to be read in the opposite way in respect of the PSNR, higher values mean lower qualities. In fact, we can see in Fig. 6 that the two lowest bit rate videos perform very well and

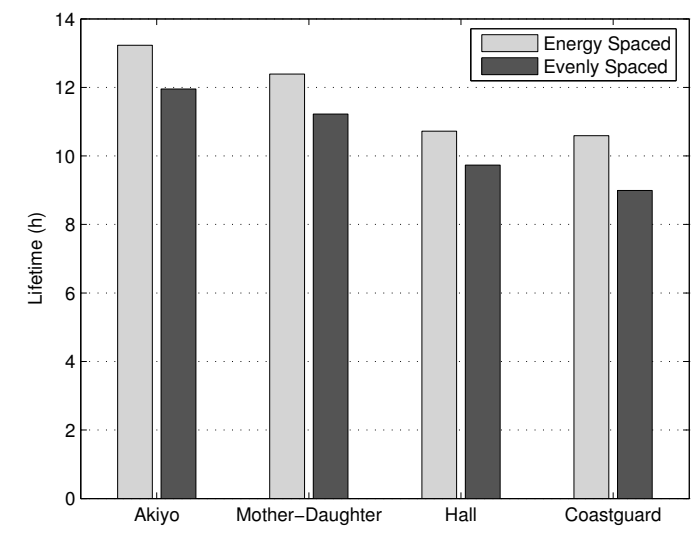

Fig. 7. Lifetime achieved by the two different placements.

that highest bit rate videos experience a slightly better quality for the evenly spaced placement $(\approx 3 \%$ and $\approx 1 \%$ for "Hall" and "Coastguard", respectively). Since the main focus of the paper is on the energy consumption of the two placements, we need to understand how much the inappreciable improvement in terms of VQM is paid back in terms of energy by the evenly spaced placement. In Fig. 7 we can see that the energy spaced placement always obtain a lifetime that is at least $10 \%$ longer than the evenly spaced scheme, and in case of "Coastguard", energy spaced outperforms evenly spaced of about $15 \%$. In absolute terms, it means that energy spaced makes multimedia nodes last from 1.5 up to 2.25 hours longer. This significant improvement in terms of energy saving supports the hypothesis to implement new energy-aware algorithms for multimedia sensor nodes based on controlled-mobility strategies.

\section{CONCLUSION}

In this paper we first evaluated the energy consumption of a feasible WMS node specifically designed to exploit the potential of the current off-the-shelf hardware technology; afterwards, we validated further the results obtained from the energy spaced placement of wireless sensor nodes by extending the analysis to multimedia traffic sources. Future research directions will address the issues related to multiflow transmission of multimedia contents on different paths throughout energy-aware routing protocols.

\section{ACKNOWLEDGMENTS}

This work is partially funded by the French National Research Agency (ANR) under the project ANR VERSO RESCUE (ANR-10-VERS-003) and the French Research National Institute of Computer Science and Automation (INRIA) under the ARC MISSION project.

\section{REFERENCES}

[1] J. K. Hart and K. Martinez, "Environmental Sensor Networks: A revolution in the earth system science?", Earth-Science Reviews (Elsevier), vol. 78, pp. 177-191, 2006. 
[2] G. Barrenetxea, F. Ingelrest, G. Schaefer and M. Vetterli, "The hitchhiker's guide to successful wireless sensor network deployments", ACM conference on Embedded network sensor systems (SenSys 2008), pp. 43 56, 2008.

[3] I.T. Almalkawi, M. Guerrero Zapata, J.N. Al-Karaki and J. MorilloPozo, "Wireless Multimedia Sensor Networks: Current Trends and Future Directions", Sensors 2010, 10, 6662-6717.

[4] T. Davis, Q. Pang, G. W. Skelton and N. Meghanathan, "On the designand implementation of wireless multimedia sensor networks", International Journal of Next-Generation Networks (IJNGN) Vol.2, No.3, September 2010.

[5] I. F. Akyildiz, T. Melodia and K. R. Chowdhury, "A survey on wireless multimedia sensor networks", Computer Networks (Elsevier), vol. 51, no. 4, pp. 921 - 960, 2007.

[6] ITU-T Recommendation, "Subjective video quality assessment methods for multimedia applications", Tech. Rep. TUT P.910, International Telecommunication Union, Geneva, Switzerland, September 1999.

[7] "Final report from the video quality experts group on the validation of objective models of video quality assessment, phase II", www.vqeg.org/.

[8] M. Cao, L. Yang, and X. Chen and N. Xiong, "Node Placement of Linear Wireless Multimedia Sensor Networks for Maximum Network Lifetime", Lecture Notes in Computer Science (Springer), vol. 5036, pp. 373-383, 2008.

[9] A. So and B. Liang, "Effect of relaying on capacity improvement in wireless local area networks", Proceedings of IEEE Wireless Communications and Networking Conference (WCNC '05), pp. 1539-1544, 2005.
[10] G.N. Shirazi and L. Lampe, "Outage optimal node placement in ultrawideband sensor networks", IEEE International Conference on UltraWideband (ICUWB), pp. 343-347, 2009.

[11] D.K. Goldenberg and J. Lin and A.S. Morse and B.E. Rosen and Y.R. Yang, "Towards mobility as a network control primitive," in Proceedings of ACM MobiHoc, 2004.

[12] E. Natalizio and V. Loscrí and E. Viterbo, "Optimal Placement of Wireless Nodes for maximizing Network Lifetime," IEEE Communications Letters, vol. 12, no. 5, pp. 1-3, May 2008.

[13] Rowing Networks wireless for less "WiFly GX" 802.11G Super Module: $\mathrm{RN}-121, \mathrm{RN}-122, \mathrm{RN}-123, \mathrm{RN}-125$. http://www.rovingnetworks.com

[14] http://www.cmucam.org

[15] A. Rowe, A. Goode, D. Goel, I. Nourbakhsh, "CMUcam3: An Open Programmable Embedded Vision Sensor," Carnegie Mellon Robotics Institute Technical Report, RI-TR-07-13 May 2007.

[16] C.H Ke, C.K. Shieh, W.S. Hwang and A. Ziviani, "An Evaluation Framework for More Realistic Simulations of MPEG Video Transmission", Journal of information science and engineering, vol. 24, no2, 2008, pp: 425-440.

[17] YUV Video Sequences - http://trace.eas.asu.edu/yuv/

[18] J. Klaue, B. Rathke, and A. Wolisz, "EvalVid - A framework for video transmission and quality evaluation", in proceedings of TOOLS, 2003, pp: 255-272.

[19] M. H. Pinson and S. Wolf, "A new standardized method for objectively measuring video quality", IEEE Transactions on Broadcasting, vol. 50, issue 3, pp: 312-322, 2004. 